NOTICE: this is the author's version of a work that was accepted for publication in the journal Diamond \& Related Materials. Changes resulting from the publishing process, such as peer review, editing, corrections, structural formatting, and other quality control mechanisms may not be reflected in this document. Changes may have been made to this work since it was submitted for publication. A definitive version was subsequently published in the journal Diamond \& Related Materials, Vol.19, (2010). DOI: http://doi.org/10.1016/j.diamond.2009.11.005 


\title{
About orientation dependence of physico-chemical properties of HPHT diamond surfaces thermally treated in $\mathrm{H}_{2}$ and $\mathrm{D}_{2}$ environments
}

\author{
Ch. Manfredotti, P. Bonino, M. De La Pierre, E. Vittone and C. Manfredotti \\ Experimental Physics Department and NIS - Nanostructures Interfaces and Surfaces - \\ Excellence Centre, University of Torino, Turin (Italy)
}

\begin{abstract}
Recently [1] we reported on some preliminary results on different physico-chemical properties of diamond (100), (110) and (111) surfaces hydrogenated by using molecular hydrogen only. The main conclusions were that thermal hydrogenation was as efficient as plasma one and that the creation of the conducting surface channel was activated by a larger energy on the (100) surface with respect to the other two. The reason, at least in the case of the comparison between (100) and (111) surfaces, could be either attributed to the presence of a carbon - oxygen double bond before hydrogenation in the former case [2] or to a better coverage by carbon - hydrogen bonds in the latter one. In the present work, further results on surface conductivity after hydrogenation steps carried out at different temperatures are described and discussed, in order to discriminate between purely thermal and kinetic effects. Moreover, other results are reported on diamond powders (0.25 micrometer mean size) in order to draw some qualitative and quantitative conclusions about hydrogen presence and behavior at the diamond surfaces. In order to better clarify the results, a large part of chemical measurements were performed after deuteration steps carried out using the same conditions as thermal hydrogenation.
\end{abstract}

\section{Introduction}

Diamond surface hydrogenation appears to be very attractive for applications in different fields, since the availability of a true transparent electrical contact on diamond is of real interest in sensors, biology, electronics, etc. $[3,4,5]$. Even if a model able to describe the creation of a surface p-channel has been existing for almost eight years, a real understanding of the physico-chemical processes leading to this situation (hydrogenation, air exposure, etc.) is still far to be reached. A possible way to simplify the situation is the 
adoption of a pure thermal approach, in order to avoid impurities, defects created by plasma, etc. and the use of high quality single crystal samples like HPHT ones. Thermal hydrogenation actually is a diamond surface catalysis due to the lower dissociation energy of hydrogen molecule with respect to $\mathrm{C}-\mathrm{H}$ bond. Different crystallographic surfaces, even reconstructed, display different kinds of bonds and, as a consequence, should have different activation energies for the creation of the surface p-channel. This is really what has been proved very preliminarily in a previous paper [1] and what will be definitively proved in this one. Bond types present at the surface are also quite important in order to describe the occurring reactions; however, it is very difficult to obtain such data by spectroscopic methods, since the p-channel layer is only $0.5 \mathrm{~nm}$ FWHM thick and particular spectroscopies such as attenuated total reflection (ATR) and similar ones are not easy to apply. Our results are a confirmation that (100) surface is much harder to be thermally hydrogenated with respect to (111) and (110) and that, most probably, only $\mathrm{CH}_{2}$ and $\mathrm{CH}_{3}$ species are present at the surface. In effect, the hydrogenation of (100) surface is impossible to observe and, moreover, values of resistivity obtained in this case after air exposure are much larger than in the case of the other two crystallographic orientations. In effect, what may be called "activation energy" for the creation of a pchannel is almost a factor of 5 higher in the case of a (100) surface.

\section{Experimental}

HPHT single crystal diamonds (IIa, dimensions $1.5 \times 1.5 \times 0.5 \mathrm{~mm}^{3}$ ), (100), (111) and (110) oriented, were supplied by Sumitomo Electric Co. Ltd. Before each hydrogenation process, in order to better remove surface contaminants and to leave a clean oxygenterminated surface, samples were oxidized using a sulphochromic acid solution at $120{ }^{\circ} \mathrm{C}$ for 4 hours, rinsed in deionized water, then in acetone and in 2-propanol, and dried in argon flow. Synthetic monocrystalline diamond powders were supplied by Advanced Abrasives Corp. (mean particle size $250 \mathrm{~nm}$, size range $0.0-0.5 \mu \mathrm{m}$, specific surface area $\mathrm{SSA}_{\mathrm{BET}}=19 \mathrm{~m}^{2} / \mathrm{g}$ ). Before hydrogenation processes, powders were treated in sulphonitric mixture 9:1 $\left(\mathrm{H}_{2} \mathrm{SO}_{4}: \mathrm{HNO}_{3}\right)$ for 1 hour at $80^{\circ} \mathrm{C}$, then washed in deionized water until neutrality, cleaned with acetone and 2-propanol and finally dried in vacuum first and then in ambient air. 
Single crystal diamonds and powders have been subjected to the same hydrogenation and deuteration processes with the following steps: samples kept in CVD reactor (high vacuum conditions, 2 hours), heating in $\mathrm{H}_{2}$ or $\mathrm{D}_{2}$ flow ( $90 \mathrm{sccm}, 1 \mathrm{~h}$ ), cooling down to $100^{\circ} \mathrm{C}$ under $\mathrm{H}_{2}$ flux ( 2 hours), chamber venting (12 hours later), removing of samples and start of electrical measurements (for 12 hours). All other measurements were carried out afterwards. Because of their small size, electrical characterization of single crystals was performed by means of a two-point probe apparatus. Other details about preparation, thermal hydrogenation of samples and measurement techniques are reported elsewhere [6].

Reflection-transmission IR spectra were obtained on a $\mu$-FT-IR PE Autoimage 2000 (32 scans for each spectrum in the $4000-700 \mathrm{~cm}^{-1}$ range, resolution $4 \mathrm{~cm}^{-1}$ ), while DRIFT spectra were obtained on a Nicolet 6700 equipped with a diffuse reflectance sphere (128 scans for each spectrum in the $4000-700 \mathrm{~cm}^{-1}$ range, resolution $4 \mathrm{~cm}^{-1}$ ).

Results and discussion

In order to build up an accurate picture of the effects induced on diamond surfaces by thermal hydrogenation, a systematic study has been carried out on three HPHT single crystal diamonds featuring different orientations, namely (100), (110) and (111). Thermal hydrogenation was investigated along a large temperature range, spanning from $600^{\circ} \mathrm{C}$ to $900^{\circ} \mathrm{C}$ in steps of $50^{\circ} \mathrm{C}$. First of all, it is worth remarking that, in the case of (110) and (111) samples, an appreciable electrical response can be found for hydrogenation temperatures of $700^{\circ} \mathrm{C}$ and above. On the other hand, in the case of (100) sample, the decay of resistivity is observable only at $850^{\circ} \mathrm{C}$ and $900^{\circ} \mathrm{C}$ : at lower temperatures this sample proved to be completely insulating.

At the end of the hydrogenation process, conducting samples showed a sheet resistivity decay after exposure to air. Such transient phenomenon shows a significant behavior within the first twelve hours; we tried to fit the corresponding experimental curves by means of an expression like:

$$
\rho(t)=a+b t^{-c}
$$

Examples of fitted resistivity transient curve for the three samples are reported in Fig. 1, while results for all the fitting procedures are given in Table 1. The reported curves 
suggest that the proposed expression provides a quite satisfying description of the experimental behavior. Nevertheless, obtained $\chi^{2}$ values, which were calculated by associating an instrumental uncertainty of $1 \mathrm{k} \Omega$ to the data points, are very high; this can be attributed to the sensitivity of the transient to the environmental conditions (in particular, humidity and $\mathrm{pH}$ ), and suggests that bigger error bars should be attributed to such process, at least of 5-6 k $\Omega$. The $a$ parameter could be interpreted as the saturation resistivity at very long times, and its values were kept fixed according to experimental curves. Coefficient $b$ represents a scaling factor for the transient, and its values change a lot along the different sessions; this behavior can be related to the wide range of starting resistivity values which was experimentally found in the different experiments. Finally, it is worth concentrating on exponent $c$, which qualitatively characterizes the behavior of the resistivity transient. All obtained values lie in a range spanning from 0.3 to 0.7 , with (100) sample featuring the highest value. This range, which is centered around a value of 0.5 , could imply some kind of diffusion or process involving a slow increase in the thickness of the surface channel, or may be also a connection between isolated p-channel regions during the exposition to air. In a "transfer doping" model [7, 8], it could well happen that radical species absorbed at the surface with their LUMO just placed in energy to accept electrons from the upward bent valence band of hydrogenated diamond may have to diffuse at the surface even at room temperature. The value of the diffusion coefficient obtained in term of building up the width or thickness of $p$ surface channel is of the order of $10^{-18} \mathrm{~cm}^{2} / \mathrm{s}$, probably not so far from the diffusion coefficient of hydrogen in diamond at room temperature $\left(10^{-20} \mathrm{~cm}^{2} / \mathrm{s}\right)$. Of course, the creation of a p-channel of about $1 \mathrm{~nm}$ over a surface of an average roughness of $20-50 \mathrm{~nm}$, likely with many defects, is a quite complex process to describe.

The final sheet resistivity values for the three samples and for each temperature are reported in Fig. 2. as a function of $1 / \mathrm{kT}$, in order to obtain some information about what may be called "activation energies" of this "transfer doping" on different surfaces. A sharp increase in resistivity may be observed in the case of (100) surface: the activation energy in this case is $1.8 \mathrm{eV}$ and it is impossible to obtain a conductivity channel at temperatures below $850^{\circ} \mathrm{C}$. In the other two cases, this is actually possible and easy for temperatures down to $700{ }^{\circ} \mathrm{C}$. The activation energies are much lower for both the (110) 
and (111) surfaces (about $0.34 \mathrm{eV}$ and $0.23 \mathrm{eV}$, respectively). Concerning (100) and (110) hydrogenated surfaces, an explanation for this phenomenon may be found in the fact that (100) oriented surface has a lower C-H bond density with respect to (110) one [9], this leading to a lower sheet carrier density and, in the end, to higher sheet resistance. However, in the case of (111) surface, it is worth remarking a clear and strong effect of desorption starting above $800{ }^{\circ} \mathrm{C}$, i.e. at the end of the hydrogenation process the surface could be less hydrogenated. These results confirm and complete those obtained previously [1] with the conclusion that (100) surface is much harder to be hydrogenated by thermal means, (111) is much easier but also less stable and, finally, (110) surface seems to be best in both terms of ease of hydrogenation and stability at the highest temperatures.

The presence of hydrogen at the surface, as measured by reflection-transmission IR, was already reported in the previous paper [1], but with some obvious difficulty and not in the case of (100) surface. In order to better prove this presence and to give more details on this aspect, we carried out hydrogenation on $0.25 \mu \mathrm{m}$ powders (see a SEM image in Fig. 3). The powders show a good deal of crystallinity, as better reported in the diffractogram displayed in Fig. 4 where standard (111), (220), (311) and (400) reflexes can be clearly identified. The reflection-transmission IR spectrum of these powders hydrogenated at $700^{\circ} \mathrm{C}$, reported in Fig. 5, shows a double series of peaks, the former being attributed to symmetrical stretching phonons $\left(\mathrm{CH}_{2}\right.$ at $2836 \mathrm{~cm}^{-1}$ and $\mathrm{CH}_{3}$ around $\left.2850 \mathrm{~cm}^{-1}\right)$, and the latter to asymmetrical ones $\left(\mathrm{CH}_{2}\right.$ around $2920 \mathrm{~cm}^{-1}$ and $\mathrm{CH}_{3}$ around $\left.2950 \mathrm{~cm}^{-1}\right)$. These attributions $[10,11,12,13]$ cannot exclude the presence of $\mathrm{C}-\mathrm{H}$ stretching modes in the same region [13] likely covered by stronger absorptions. In fact, looking at Fig. 6 where DRIFT spectra of hydrogenated powders at different temperature are reported, it must be noticed that the peak at $2870 \mathrm{~cm}^{-1}$, that can be attributed to C-H stretching mode on (111) surface [10], grows faster as a function of temperature with respect to the peak at 2837 $\mathrm{cm}^{-1}$ and that the peak at $2925 \mathrm{~cm}^{-1}$, which can be attributed to $\mathrm{C}-\mathrm{H}$ stretching mode at (100) surface, is not present in the spectrum at $600^{\circ} \mathrm{C}$ and grows much faster with temperature with respect to peaks around $2950 \mathrm{~cm}^{-1}$ at $700^{\circ} \mathrm{C}$. At the same time, the peaks in the $1600-1800 \mathrm{~cm}^{-1}$ region, likely due to $\mathrm{C}=\mathrm{O}$ stretching mode in different chemical environments, show a noticeable decrease. This shows that hydrogen 
progressively substitutes oxygen at the surface and that, quite likely, the former $\mathrm{C}-\mathrm{H}_{2}$ or $\mathrm{C}-\mathrm{H}_{3}$ bonds created at lower temperatures are progressively substituted by $\mathrm{C}-\mathrm{H}$. Moreover, comparing the behavior of the two groups of peaks, the importance of the group at larger energies that should be attributed to stretching modes at (100) surface, becomes progressively comparable with the lower energy group, due to stretching modes at (111) surface. Higher hydrogenation temperatures might be required in order to reveal $\mathrm{C}-\mathrm{H}$ (100) stretching modes, due to the presence of C-O-C ether-like structures at the surface, that are reported to be more stable than other oxygen containing groups as well as favorable configurations for the $(100)$ orientation $[14,15]$. Unfortunately, the wide overlapping of different absorptions in the low frequency region of the DRIFTS spectra doesn't allow individuating clearly C-O stretching bands and their modifications with hydrogenation temperature. The slower conversion in the case of the presence of C-O-C groups must anyway be confirmed, as other theoretical calculations [16] indicate that the stability of the bridge ether with respect to on-top ketone one depends on lattice parameters and that it increases as the cell parameters decrease. This is in agreement with the results shown in Fig. 2, which indicated that the creation of the conducting sheet at (100) surface is energy activated with respect to (111), which displays an activation energy close to zero.

In order to discriminate against different kinds of hydrogen contamination, powders deuteration was carried out in the same conditions of hydrogenation at 600, 700 and $800^{\circ} \mathrm{C}$. On these powders the relative absorbance was measured according to the same Kubelka-Munk method by diffused reflectance FTIR or DRIFT and the relevant results are shown in Fig. 7, together with the spectrum of an oxidized powder sample as reference. The C-D stretching modes around $2180 \mathrm{~cm}^{-1}[11,12,13]$, not present in the oxidized sample, increase with treatment temperature, with some saturation between $700^{\circ} \mathrm{C}$ and $800^{\circ} \mathrm{C}$, while, at the same time, $\mathrm{C}=\mathrm{O}$ stretching modes nearby progressively disappear. These results are in agreement with those ones obtained on single crystal diamond by a standard MWCVD hydrogenation/deuteration process [11]. In the same spectra it is possible to observe the progressive decrease of water content by the increasing hydrophobicity due to deuterium attachment at the surface. The observation of a splitting in C-D modes is in agreement with the consideration that, as described before, 
while the peak at $2180 \mathrm{~cm}^{-1}$ is attributed to a C-D stretching mode normal to the (100) surface [12], the other peak around $2130 \mathrm{~cm}^{-1}$ could be attributed to a stretching mode normal to (111) surface [10]. In fact, as in the hydrogenation case, the former seems to increase faster as a function of temperature with respect the latter one. In conclusion, it seems reasonable to state that a pure thermal hydrogenation process occurs preferably at lower temperatures on surfaces with single dangling bonds, even if defects could play an important role in the hydrogenation itself [10].

\section{Conclusions}

Thermal hydrogenation of diamond, carried out in absence of damaging effects of hydrogen plasma, gives rise to a surface conducting layer with the same electrical characteristics observed in plasma hydrogenation, but shows a strong dependence on crystallographic direction. While for (111) and (110) surfaces hydrogenation may be carried out at any temperature between $700{ }^{\circ} \mathrm{C}$ and $900^{\circ} \mathrm{C}$, similar results are obtained only above $800^{\circ} \mathrm{C}$ for (100) surfaces. The time dependence of conducting sheet creation after exposure to air has been accurately followed and it seem to show a square root dependence, with a clear indication of the presence of some diffusion related process which may be related to the enlargement of conducting hydrogenated "islands" (starting from defects?) and the progressive elimination of oxygen. This is clearly seen both in hydrogenated and deuterated powders where $\mathrm{C}=\mathrm{O}$ bonds decrease at increasing treatment temperatures is accompanied by the progressive growth of $\mathrm{C}-\mathrm{H}$ or $\mathrm{C}-\mathrm{D}$ related peaks. This process needs higher energies and its occurrence is more difficult in the case of (100) surface, since the oxygen at the surface may be bonded in a more stable ether-like configuration, which is characteristic of (100) orientation and can slower the conversion to a hydrogen terminated surface. Moreover, this behavior may be related to the fact that a simple C-H bond at (100) surface is stronger than $\mathrm{C}-\mathrm{H}_{2}$ or $\mathrm{C}-\mathrm{H}_{3}$ bonds on (111) and possibly also on (110) surfaces and therefore it becomes more important at higher temperatures. 
References

1) C. Manfredotti, F. Fizzotti, A. Lo Giudice, Ch. Manfredotti, M. Castellino, P. Bonino and E. Vittone, Diamond Relat. Mater. 17 (2007), 1154

2) P. John, N. Polwart, C. E. Troupe and J. I. B. Wilson, J. Am. Chem. Soc. 125 (2003), 6600

3) P. Ariano, P. Baldelli, E. Carbone, A. Gilardino, A. Lo Giudice, D. Lovisolo, C. Manfredotti, M. Novara, H. Sternschulte, E. Vittone, Diam. Relat. Mater. 14 (2005), 669

4) M. Tachiki, Y. Kaibara, Y. Sumikawa, M. Shigeno, T. Banno, K. S. Song, H. Umezawa, H. Kawarada, Phys. Stat. Sol. A Appl. Res. 199 (2003), 39

5) C. E. Nebel, H. Kato, B. Rezek, D. Shin, D. Takeuchi, H. Watanabe, T. Yamamoto, Diam. Relat. Mater. 15 (2006), 264

6) F. Fizzotti, A. Lo Giudice, Ch. Manfredotti, C. Manfredotti, M. Castellino, E. Vittone, Diam. Relat. Mater. 16 (2007), 836

7) J. Ristein, Science 313 (2006), 1057

8) S. J. Sque, R. Jones, S. Oeberg, P. R. Briddon, Physica B 376-377 (2006), 268

9) K. Hirama, H. Takayanagi, S. Yamauchi, J. H. Yang, H. Kawarada and H. Umezawa, Appl. Phys. Lett. 92 (2008), 112107

10) K. Ushizawa, M. Gamo, Y. Kikuchi, I. Sakaguchi, Y. Sato and T. Ando, Phys. Rev. B 60 (1999), R5165

11) T. Ando, M. Ishii, M. Kamo and Y.Sato, Diam. Rel. Mat. 4 (1995), 607

12) L. Ley, B. F. Mantel, K. Matura, M. Stammler, K. Janischowsky and J. Ristein, Surf. Sci. 427-428 (1999), 245

13) Y. C. Sun and J. M. Chen, J. Phys. Chem. B 101 (1997), 7082

14) S. J. Sque, R. Jones and P. R. Briddon, Phys. Rev. B 73 (2006), 085313

15) Y. M. Wang, K. W. Wong, S. T. Lee, M. Nishitani-Gamo, I. Sakaguchi, K. P. Loh and T. Ando, Diam. Relat. Mater. 9 (2000), 1582

16) H. Tamura, H. Zhou, K. Sugisako, Y. Yokoi, S. Takami, M. Kubo, K. Teraishi, A. Miyamoto, A. Imamura, M. N. - Gamo and T. Ando, Phys. Rev. B 61 (2000), 11025 
Figure captions

Table 1: List of the results of the fitting sessions performed over the experimental curves of the resistivity decay obtained at different temperatures for the three HPHT samples.

Figure 1: Fitted surface resistivity decay curves obtained for the three HPHT samples in the case of a thermal hydrogenation process at $900^{\circ} \mathrm{C}$.

Figure 2: Arrhenius plot of final surface resistivity as a function of $1 / \mathrm{kT}$, measured on the three HPHT samples thermally hydrogenated at different temperatures. The experimental $1 / \mathrm{kT}$ range corresponds to a temperature range $700^{\circ} \mathrm{C}-900^{\circ} \mathrm{C}$.

Figure 3: SEM image of $0.25 \mu \mathrm{m}$ diamond powder

Figure 4: XRD spectrum of $0.25 \mu \mathrm{m}$ diamond powder

Figure 5: Reflection-transmission IR spectra of $0.25 \mu \mathrm{m}$ diamond powder thermally hydrogenated at $700{ }^{\circ} \mathrm{C}$, recorded on different points of the sample

Figure 6: DRIFT spectra of $0.25 \mu \mathrm{m}$ diamond powder thermally hydrogenated at 600 , 700 and $800{ }^{\circ} \mathrm{C}$. The spectrum of oxidized diamond powder is shown for comparison purposes. Spectra are shifted only for visualization purposes.

Figure 7: DRIFT spectrum of $0.25 \mu \mathrm{m}$ diamond powder thermally deuterated at 600 , 700 and $800{ }^{\circ} \mathrm{C}$. The spectrum of oxidized diamond powder is shown for comparison purposes. 